\title{
Reparative effects of neural stem cells in neonatal rats with hypoxic-ischemic injury are not influenced by host sex
}

\author{
Stephen Ashwal', Nirmalya Ghosh'1, Christine I.Turenius' ${ }^{1}$ Melissa Dulcich², Christopher M. Denham', Beatriz Tone', \\ Richard Hartman', Evan Y. Snyder ${ }^{3}$ and Andre Obenaus ${ }^{1,4}$
}

\begin{abstract}
BACKGROUND: Gender is increasingly recognized as an important influence on brain development, disease susceptibility, and response to pharmacologic/rehabilitative treatments. In regenerative medicine, it remains entirely unknown whether there is an interaction between transplanted stem cells and host gender that might bias efficacy and safety in some patients but not others.
\end{abstract}

METHODS: We examined the role of recipient gender in a neonatal rat hypoxic-ischemic injury (HII) model, treated with female human neuronal stem cells (hNSCs), labeled with superparamagnetic iron oxide particles implanted into the contralateral cerebral ventricle. We monitored HII evolution (by magnetic resonance imaging, histopathology, behavioral testing) and hNSC fate (migration, replication, viability).

RESULTS: Recipient gender after implantation did not influence the volume or location of ischemic injury $(1,30$, or $90 \mathrm{~d}$ ) or behavior (90 d). Superparamagnetic iron oxide labeling did not influence HII evolution. Implantation had its greatest benefit on mild/moderate injuries, which remained stable rather than increasing as in severe HII as is the natural history for such lesions. CONCLUSION: Our results suggest that hNSC treatment (including using hNSCs that are prelabeled with iron to allow tracking in real time by magnetic resonance imaging) would be equally safe and effective for male and female human newborns with mild-to-moderate HII.

G ender is increasingly recognized to play an important role in adult and pediatric neurologic disease (1). The relation to outcome is complex as it is associated with at least four primary variables: (i) effects on brain development (2), (ii) specific biological susceptibility to disease (3-6), (iii) genderdependent ability of the nervous system to respond to pharmacologic or rehabilitative treatments (7-11), and (iv) gender of donor cells and their interactions with host tissues in individuals undergoing transplantation $(12,13)$.

Accumulating clinical and translational evidence suggests that the male neonatal brain is more susceptible to hypoxic-ischemic injury (HII) $(1,5,6)$. While the underlying mechanisms remain elusive, it is likely that gonadal hormones and differences in gender-dependent gene expression modulate many of the cellular reactions. Similarly, compelling data suggest that the female neonatal brain responds better to HII neuroprotective treatments $(1,5,6,9)$.

The importance of gender effects from both donor cells and on host tissues is recognized in the field of transplantation (12-14) and in view of the strong interest in pursuing stem cell treatment for neonatal HII $(15,16)$, we examined the effect of host gender on the outcome of such treatment. Our study evaluated the role of recipient gender in a rat pup model of neonatal HII after human neuronal stem cells (hNSC) implantation. Using a model of unilateral permanent carotid occlusion followed by $8 \%$ hypoxia in both male and female rat pups, we implanted female hNSCs, labeled with and without superparamagnetic iron oxide (SPIO) particles, into the lateral ventricle contralateral to the injury and analyzed (i) progression of HII and (ii) therapeutic activity and fate of implanted hNSCs, including replication and viability. HII progression was determined by (i) serial high-field magnetic resonance imaging (MRI), (ii) neurological testing that measured motor impairment, (iii) behavioral testing that assessed functional outcome, and (iv) histology and immunohistochemistry.

\section{RESULTS}

\section{Animal Weights and Neurological Scores}

No significant weight differences between groups were found, although post-HII weights were always lower for females. Compared to mildly injured animals at $90 \mathrm{~d}$ post-HII $(323 \pm 26 \mathrm{~g})$, moderately injured animals were $3.45 \%$ lighter $(312 \pm 13 \mathrm{~g})$ and severely injured animals were $18.65 \%$ lighter (272 $\pm 24 \mathrm{~g})$. Weights on days 1-4 post-HII were negatively correlated with MRI lesion volumes at 1,30 , and $90 \mathrm{~d}$ post-HII (lower weight associated with larger lesion; $r=-0.3$ to -0.54 ; $P<0.05)$. No overall differences (i.e., at $90 \mathrm{~d}$ ) in mortality rates were observed for male (60\%) compared to female (50\%) rat pups (50 males, 50 females).

Total neurological scores and righting reflex times were significantly different between injury severity groups at $1 \mathrm{~d}$ but

\footnotetext{
'Department of Pediatrics, Loma Linda University School of Medicine, Loma Linda, California; '2Department of Psychology, Loma Linda University School of Behavioral Health, Loma Linda, California; ${ }^{3}$ Sanford-Burnham Medical Research Institute, Program in Stem Cell \& Regenerative Biology, La Jolla, California; ${ }^{4}$ Division of Translational Studies, School of Behavioral Health, Loma Linda University School of Medicine, Loma Linda, California. Correspondence: Stephen Ashwal (sashwal@llu.edu) 
not at $2 \mathrm{~d}$ post-HII. The righting reflex time at $1 \mathrm{~d}$ post-HII correlated with weight at $2 \mathrm{~d}$ post-HII $(P<0.05)$. At $48 \mathrm{~h}$, righting took 10 and $17 \%$ longer in moderately and severely injured as compared to mildly injured animals. In moderately injured animals, there were no neurological or behavioral differences based on gender or iron labeling.

\section{MRI-Based Lesion and hNSC Volumes}

We found no differences in the volume or location of ischemic brain injury on MRI for male vs. female pups or labeled vs. unlabeled hNSCs (Figure 1a). Hierarchal region splitting derived lesion volumes serially increased for all severities (classified by rat pup scoring system (RPSS); Figure 2), particularly in severely injured animals (Figures $1 \mathrm{~b}$ and $3 \mathrm{c}$ ), as we have previously established is the natural history of HII lesions (17). Female pups had smaller HII volumes than male pups (Figure 3b,d) but were not significantly different.

Transplanted hNSCs were found in the ipsilateral ventricle, on the side of implantation, at $1 \mathrm{~d}$ postimplantation and were detected on the contralateral side (adjacent to the HII lesion) at 30 and $90 \mathrm{~d}$ (Figures $1 \mathrm{~b}$ and $4 \mathrm{~b}$ ), suggesting extensive migration and "pathotropism." 3D reconstructions confirmed the unilateral nature of the HII lesion and the bilateral distribution of the hNSCs in animals from all severity groups

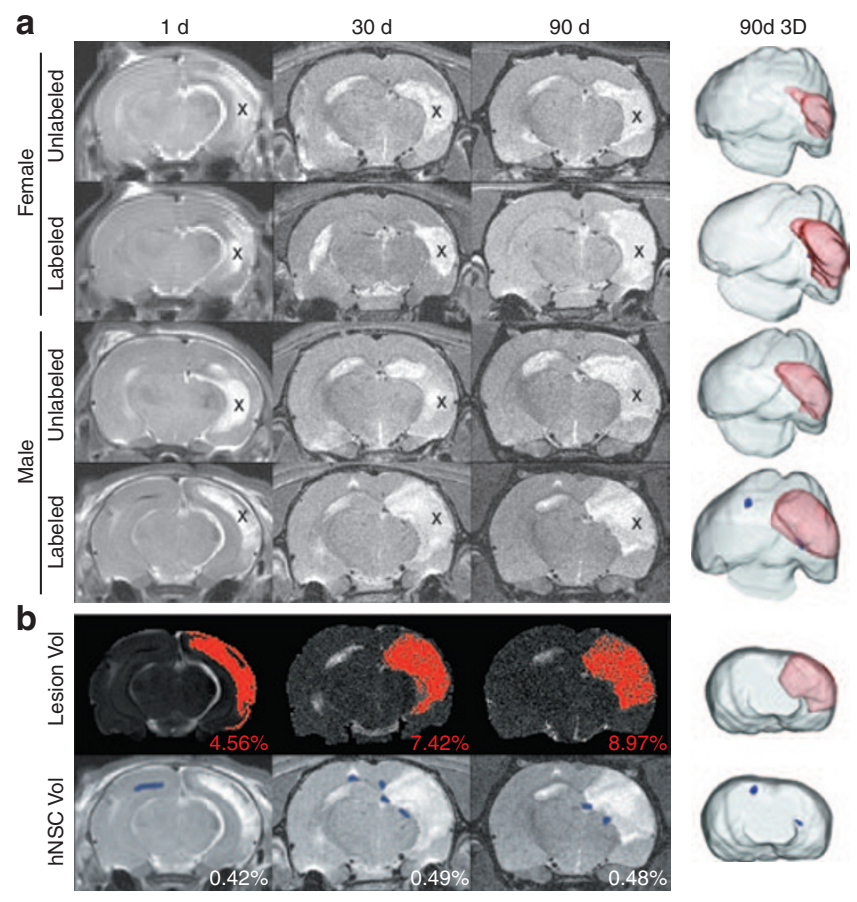

Figure 1. Neither gender nor labeling status of hNSC alters lesion volumes. (a) No significant effects were observed at any time point when lesion volume was assessed for gender (male vs. female) or for whether hNSCs were SPIO labeled/unlabeled. 3D lesion volume reconstruction (90 d) illustrates this lack gender or labeling status. (b) HRS was used to extract HII volumes from T2WI (same male/labeled animal shown in panel a, bottom). hNSC volumes were similarly extracted. Reconstructions show the extent of the lesion (red) and hNSC volumes (blue). X indicates lesion. HII, hypoxic-ischemic injury; hNSC, human neuronal stem cells; HRS, hierarchal region splitting; SPIO, superparamagnetic iron oxide; $\mathrm{T} 2 \mathrm{WI}$, T2-weighted.
(Figure 1a,b). We have previously published an MRI algorithm by which hNSC "volume" can be translated into the degree of hNSC migration and proliferation (17).

\section{RPSS and Lesion Volumes}

RPSS scores were significantly different between groups $(P<0.001$; Table 1). Gender and hNSC labeling did not affect RPSS (Table 2). There was a significant $(P<0.001)$ correlation between RPSS and lesion volume at $1 \mathrm{~d}$, but the correlation was weaker at 30 and $90 \mathrm{~d}$ (Figure 2).

\section{Lesion Location and Size}

The distribution, location, and size of the HII lesions were similar among moderately injured animals on MRI and confirmed by cresyl violet histology (Figure 3a). MRI and histologically stained brains at $90 \mathrm{~d}$ were matched in lesion size and morphology. Neither MRI nor histological analysis showed an effect of gender or iron labeling on HII lesion volumes at $90 \mathrm{~d}$ (Figure 3a,b). In contrast to untreated animals, lesion volumes in hNSC-transplanted rats were stable at 1,30 , and $90 \mathrm{~d}$ in mildly $(0.7,0.9$, and $0.6 \%$ of total brain volume) and moderately (5.4, 5.6, and 7.5\%) injured animals. Despite hNSC implantation, lesion volume significantly increased from $1 \mathrm{~d}$ to $30 \mathrm{~d}$ to $90 \mathrm{~d}$ in severely injured animals $(12.7,19.4$, and $25.2 \%$; Figure $3 c$ ).

\section{hNSC Volumes and Viability}

We found greater hNSC volumes in males than in females $(P<$ 0.001 ) at $30 \mathrm{~d}$, when these measures peaked in both groups, but not at 1 or $90 \mathrm{~d}$ (Figure 4a). There was a significant $(P<0.001)$ increase in hNSC volume over time on the implanted uninjured side but not on the injury side (Figure $4 \mathrm{~b}$ ). No significant gender effect was seen between hNSC volumes and HII lesion volume at $90 \mathrm{~d}$ (Figure 4c). No cell overgrowth, tumor formation, deformation, or abnormalities were noted.

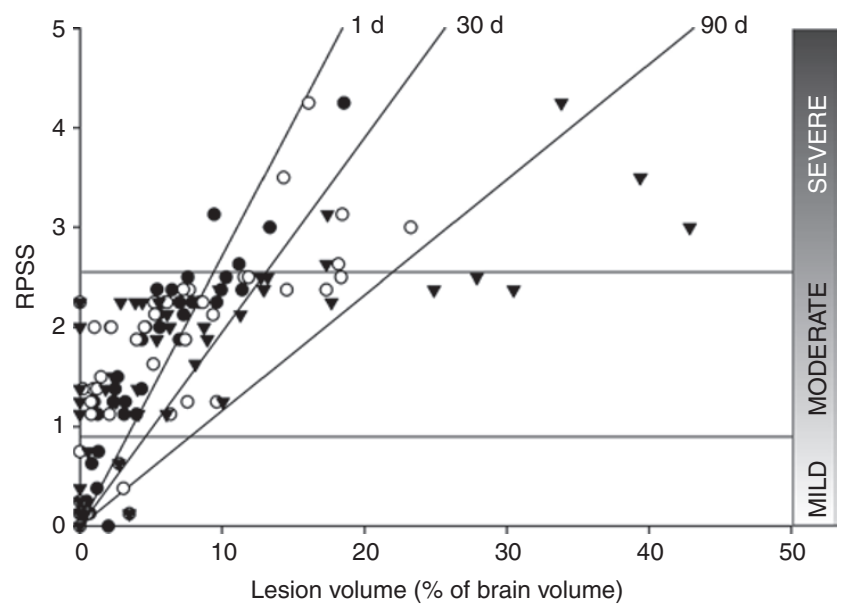

Figure 2. Manual RPSS and semiautomated HRS produce comparable lesion volumes. RPSS at $1 \mathrm{~d}$ postimplantation categorized animals based on injury severity. HRS correlated well with the RPSS method $\left(R^{2}: 1 \mathrm{~d}=\right.$ $0.813,30 d=0.550,90 d=0.543$ ). Filled circles indicate HRS $1 d$; open circles indicate HRS $30 \mathrm{~d}$; inverted triangles indicate HRS $90 \mathrm{~d}$. HRS, hierarchal region splitting; RPSS, rat pup scoring system. 
a Male unlabeled

Male SPIO

Female unlabeled

Female SPIO
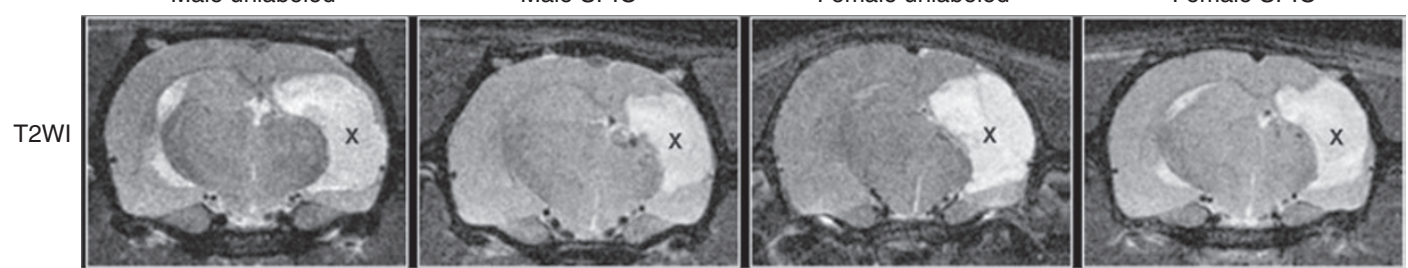

CV
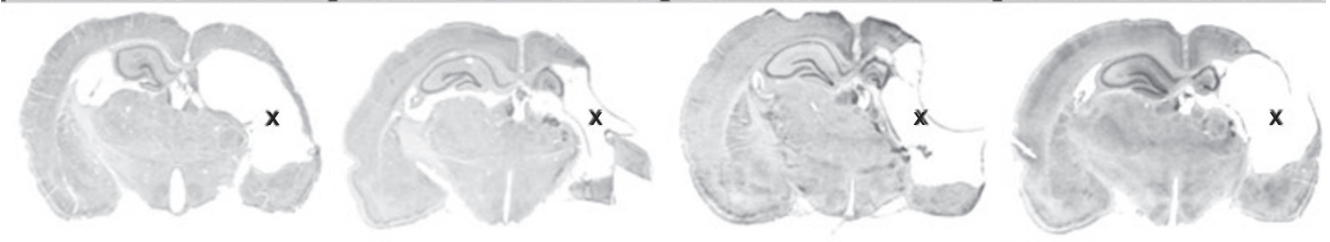

b

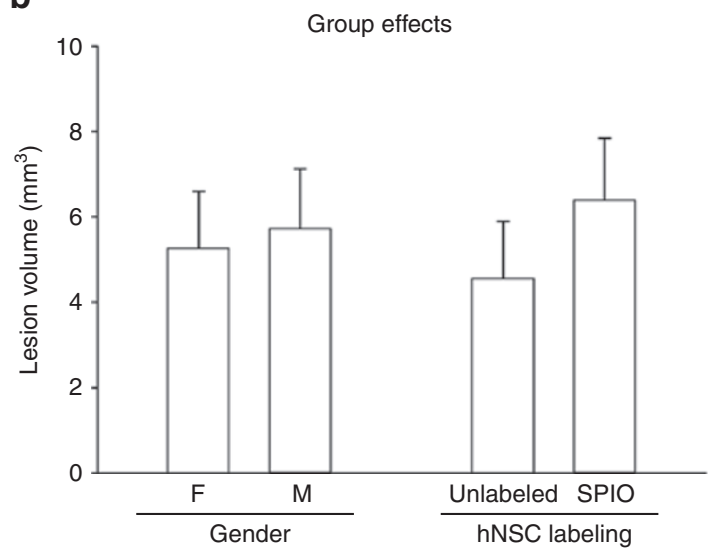

C

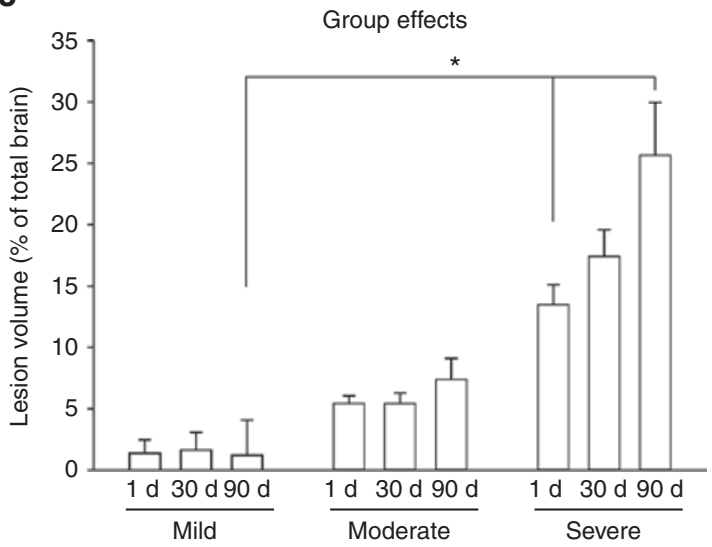

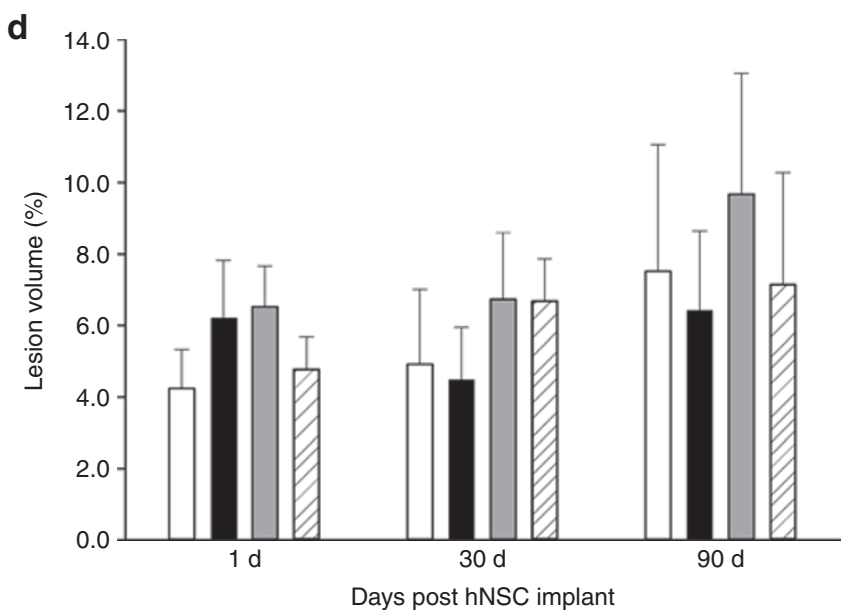

Figure 3. Lesion volumes are not influenced by gender or hNSC labeling. (a) At $90 \mathrm{~d}$, post-HII T2WI lesions (hyperintensities, X) appeared similar across all gender and implant groups. Cresyl violet (CV) postmortem histology (lower panel) showed similar lesion size to T2WI (upper panel). Cystic lesions (X) were similar between gender- and hNSC-labeled animals; (b) lesion volumes are not altered by gender or labeling; (c) lesion volume evolution over time did not change in mildly injured animals, but there was an increase in lesion volume in moderate and severe HII injury groups (see text). Across all groups, there was a significant difference between severity groups at 1 and $90 \mathrm{~d}(P=0.05)$. (d) No significant differences were found in lesion volumes over the 90 d experimental period by gender or labeling. (Comparison of gender, labeling status, and lesion volume, $P=0.684$; white bar: male unlabeled; black bar: female unlabeled; gray bar: male SPIO; hatched bar: female SPIO). HII, hypoxic-ischemic injury; hNSC, human neuronal stem cells; SPIO, superparamagnetic iron oxide; $\mathrm{T} 2 \mathrm{WI}, \mathrm{T} 2$-weighted.

\section{Immunohistochemistry}

Our immunohistological data corroborated our MRI findings that neither gender nor hNSC labeling status altered ischemic injury or hNSC volumes. Human glial fibrillary acidic protein (hGFAP) immunoreactive cells exhibiting classical astrocyte morphology were consistently found in the striatum (Figure $5 a)$, with the most robust expression observed along the third ventricle adjacent to the ventral hypothalamus (Figure $5 \mathrm{~b}$ ), exhibiting morphology similar to radial glial cells often proximal to the ventricle (Figure 5b, expanded). 

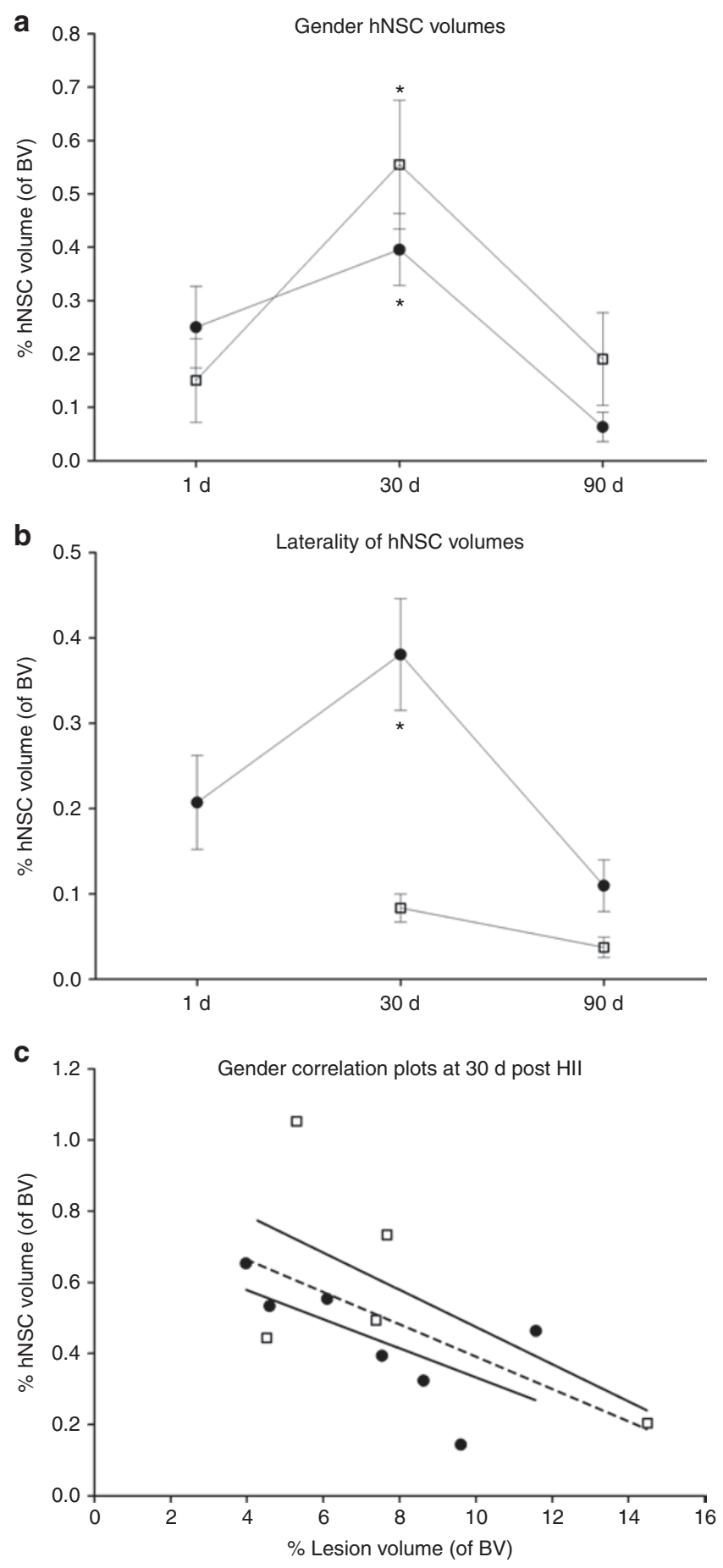

Figure 4. Labeled hNSCs had no effect on gender or lesion volumes in moderate HII. (a) There were no significant differences between hNSC volumes extracted from MRI neither between males (filled circles) or females (open squares) nor between time points $(1,30,90 \mathrm{~d} ; P=0.099)$. However, a highly significant difference in hNSC volumes was found at 30 $\mathrm{d}\left({ }^{*} P=0.00005\right)$ compared with 1 or $90 \mathrm{~d}$ post-HII. (b) We also observed a significant difference in hNSC volumes between the implanted side (left, filled circles) and the hNSC volumes observed on MRI on the injury side (right; open squares, ${ }^{*} P=0.0006$ ), but no differences were seen between males and females $(P=0.172)$. (c) At $30 \mathrm{~d}$ post-HII, male and female correlation plots of hNSC compared with HII lesion volumes did not reveal any significant interactions (males: open squares, $R^{2}=0.41$; females: filled circles, $R^{2}=0.44$ ). These results further confirmed that iron labeling of hNSC was not influenced by gender resulting in similar lesion volumes
Table 1. Injury severity group assignments

\begin{tabular}{lccccc} 
Injury severity & $\begin{array}{c}\text { Male } \\
\text { labeled }\end{array}$ & $\begin{array}{c}\text { Male } \\
\text { unlabeled }\end{array}$ & $\begin{array}{c}\text { Female } \\
\text { labeled }\end{array}$ & $\begin{array}{c}\text { Female } \\
\text { unlabeled }\end{array}$ & RPSS $^{\text {b }}$ \\
\hline Mild & 0 & 5 & 0 & 4 & $0.31 \pm 0.09$ \\
Moderate & 6 & 7 & 8 & 8 & $1.80 \pm 0.10$ \\
Severe & 1 & 1 & 4 & 1 & $3.07 \pm 0.24$ \\
\hline
\end{tabular}

Data show the number of rat pups/group for different injury severity groups,

categorized by gender and whether they were implanted with SPIO-labeled human neuronal stem cells.

RPSS, rat pup scoring system; SPIO, superparamagnetic iron oxide.

aBased on RPSS. ${ }^{\text {AAll }}$ animals combined by group severity.

Table 2. RPSS comparisons for injury severity, gender, and hNSC labeling status

\begin{tabular}{lccc}
\hline Injury severity & Female & Male & $P^{\text {a }}$ \\
\hline Mild & $0.32 \pm 0.12$ & $0.31 \pm 0.15$ & 0.960 \\
Moderate & $1.85 \pm 0.13$ & $1.73 \pm 0.15$ & 0.548 \\
Severe & $3.15 \pm 0.33$ & $2.88 \pm 0.25$ & 0.654 \\
Moderate labeled & $1.84 \pm 0.20$ & $2.00 \pm 0.19$ & 0.584 \\
Moderate unlabeled & $1.86 \pm 0.18$ & $1.53 \pm 0.19$ & 0.228 \\
\hline
\end{tabular}

hNSC, human neuronal stem cells; RPSS, rat pup scoring system.

at-Test comparisons.

Human nestin (hNestin), a marker of undifferentiated neural progenitors, in donor-derived cells located in the hemisphere contralateral to the injury was typical of intermediate filament proteins (Figure 5c). hNestin expression was abundant in the lining of the ventricles (a region lined by a NSC germinal zone) and next to the lesion (Figure 5d, expanded), but absent within the parenchyma. The hNestin expression within cells along the ventricular lining was reminiscent of tangles arising from a central core, as seen in some glial morphologies. However, hNestin and hGFAP did not colocalize in the parenchyma or along the ventricles (Figure $5 \mathrm{e}-\mathrm{g}$ ).

Rodent GFAP staining (i.e., marking host cells) was prominent next to the lesion and within the tissue parenchyma (Figure 5h, expanded); hGFAP was expressed, within glial processes throughout the parenchyma (Figure 5i, expanded). hGFAP somata were consistently found in or near the corpus callosum (Figure 5f), ipsilesional cortex (Figure 5i), and striatum (Figure 5a). hGFAP-labeled astrocytes (Figure 5j) were found embedded adjacent to native rat astrocytes (Figure 5i, expanded) but were never found to colocalize with these native cells (Figure 5j, expanded).

\section{Behavioral Outcomes}

Behavioral assessment just prior to the final $90 \mathrm{~d}$ time point found no significant differences based on gender or hNSC labeling (Figure 6). Neither gender nor labeling had any effects on anxiety-like behaviors in the elevated zero maze, although

in each group over time. However, when male and female cohorts were combined, a significant correlation (dotted line, $R=0.585 ; P=0.046$ ) was found demonstrating that increased hNSC volumes were associated with smaller HII lesion volumes, irrespective of gender. BV, brain volume; $\mathrm{HII}$, hypoxic-ischemic injury; hNSC, human neuronal stem cells; MRI, magnetic resonance imaging. 
females spent more time in the enclosed arms, suggesting some increased anxiety $(P<0.02$; data not shown).

Mildly injured animals exhibited superior spatial memory during water maze probe trials compared to moderately and severely injured animals $(P<0.007)$, independent of gender or hNSC labeling status (Figure 6a). When labeling and gender were pooled for a global analysis of lesion size on behavior, we found that mild and moderate HII animals habituated to

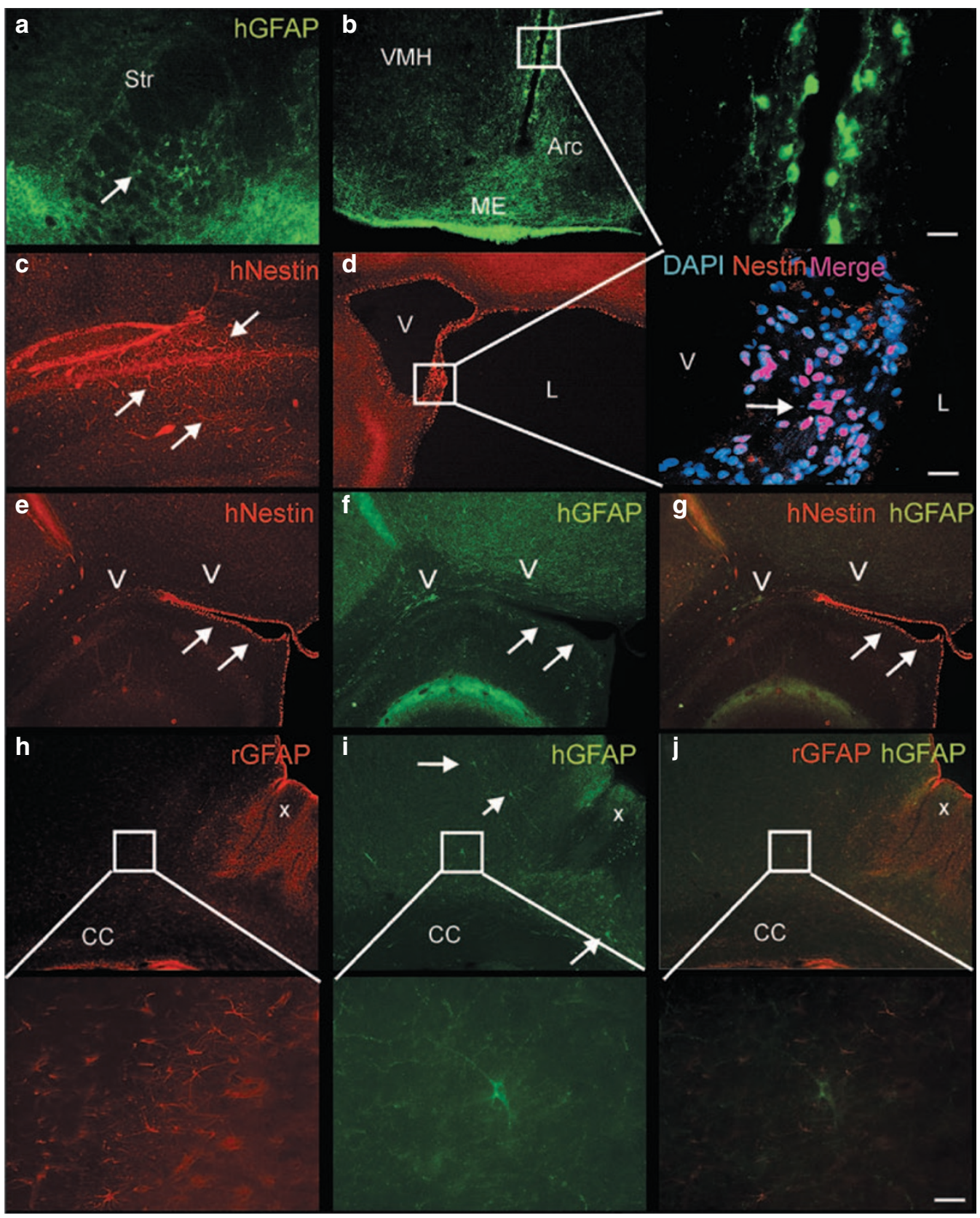

Figure 5. Immunostaining for hNSC reveals immature and mature cellular phenotypes independent of gender or hNSC labeling status.

(a) Immunostaining for human GFAP (hGFAP) identified positive (+) cells in the ipsilateral striatum (Str) adjacent to the lesion that had a mature astrocytic morphology distinct from those seen adjacent to the third ventricle. (b) hGFAP+ ${ }^{+}$cells were also observed within the periventricular region, the median eminence (ME), and the arcuate nucleus (Arc), with fewer hGFAP+ cells were seen in the ventro-medial hypothalamus (VMH). hGFAP ${ }^{+}$cells lining the third ventricle had similar morphology to astrocytic radial glia. (c) Human nestin (hNestin) within the contralateral cortex revealed hNSC that exhibited an intermediate filament morphology (arrows). (d) In the ipsilateral hemisphere, hNestin staining was observed adjacent to the ventricle (V), as well as tissues exposed to cerebrospinal fluid, such as cystic regions of the lesion (L), consistent with a more immature cellular phenotype, with only 50\% of these cells nestin+. (e-g) hNestin ${ }^{+}$staining (arrows) along the ventricles did not colocalize with hGFAP+ cells (arrows). (h) Abundant endogenous rodent (rGFAP) astrogliosis (X) was observed in the ipsilateral cortex adjacent to the HII lesion. Higher magnification revealed numerous rGFAP+ astrocytes. (i) In contrast, only scattered hGFAP ${ }^{+}$cells were observed within the cortical regions adjacent to the HII lesion. High magnification illustrated typical astrocyte morphology in an hGFAP+ cell. (j) No colocalization of human (green) and rat (red) astrocytes was observed. Higher magnification illustrates a single human astrocyte (green) surrounded by rodent astrocytes (red) with no colocalization. Bar $=20 \mu \mathrm{m}$. DAPI, 4',6-diamidino-2-phenylindole; GFAP, glial fibrillary acidic protein; HII, hypoxic-ischemic injury; hNSC, human neuronal stem cells. 

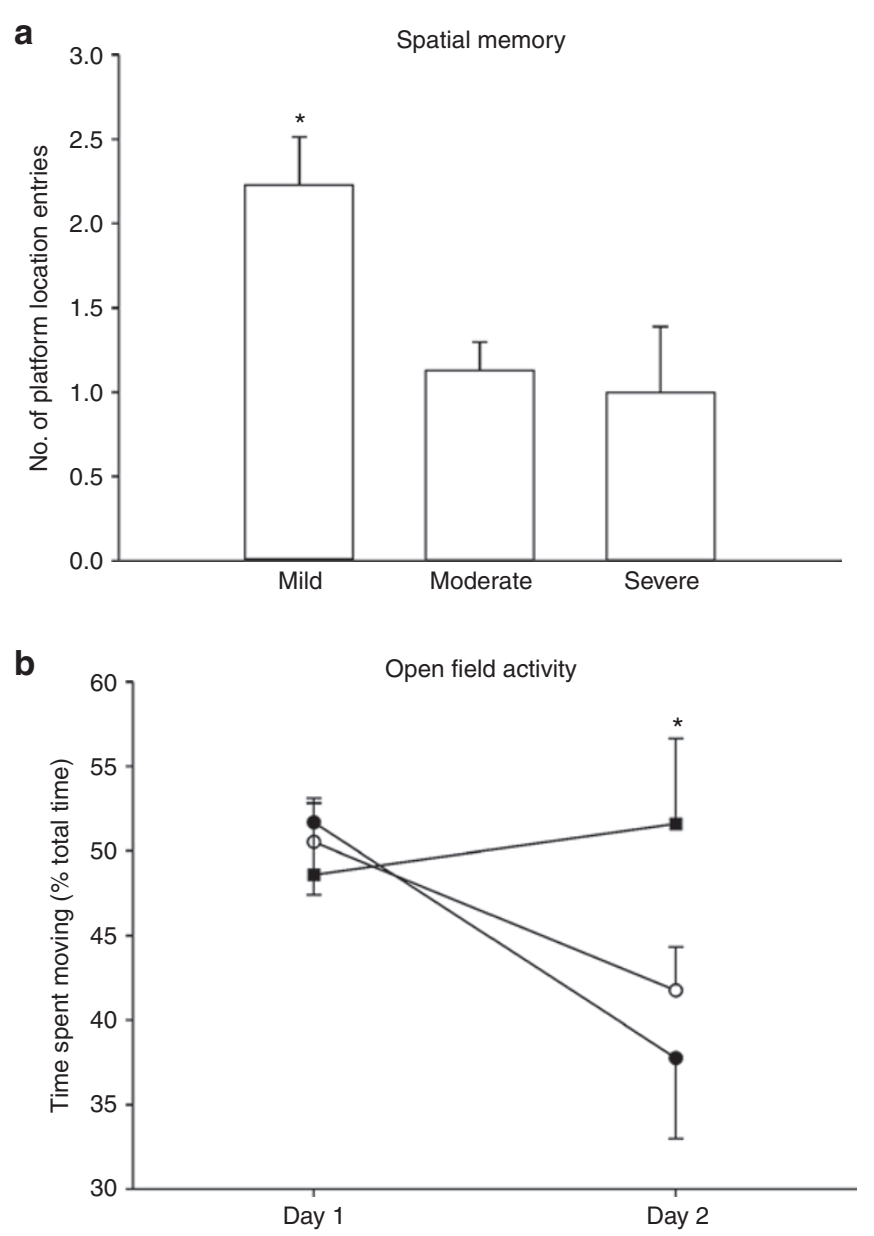

Figure 6. Behavioral testing demonstrated learning and exploratory deficits based on injury severity but not on gender or labeling status. (a) Behavioral testing based on lesion severity revealed that those animals with mild injury had improved spatial memory compared to moderately or severely injured animals $(P<0.007)$. (b) Similarly, animals with severe injury spent more time exploring an open field than their mild and moderate injury counterparts $(P<0.04)$. Filled circles indicate mild injury; open circles indicate moderate injury; filled squares indicate severe injury.

the open field with significantly decreased exploratory behavior during their second exposure to the open field, whereas severely injured animals did not $(P<0.04$; Figure $6 \mathbf{b})$. Thus, behavioral evaluation discriminated between injury severities but not gender or hNSC labeling status.

\section{DISCUSSION}

Our study revealed three clinically relevant and important findings: (i) HII recipient gender of an hNSC implant does not affect the volume or location of ischemic injury at 1-90 d postinjury or ultimate behavioral outcomes, (ii) hNSC iron labeling did not affect HII volume or behavior, and (iii) lesion volumes in pups receiving hNSCs remained stable and did not increase in size in mild and moderate severity groups, but such a beneficial effect was not seen in the severely injured group where lesion volume continued to increase. The reparative efficacy of implanted hNSC compared to no implantation control groups has been previously discussed by us and others in published works $(18,19)$. The focus of our current study was to evaluate whether host gender may impact that efficacy in the neonatal brain (as interventions often do in adult stroke) and to assess the risk of toxicity to the developing brain from the iron labeling required to track implanted stem cells by MRI as will be required in any clinical trial addressing perinatal hypoxic-ischemic encephalopathy.

\section{Gender Effects}

Gender has proven important in four broad areas affecting the developing nervous system, prompting our studies. Gender effects on brain development have been reviewed, with the suggestion that anatomical differences may, explain differences in disease occurrence and susceptibility (2). This has been noted in males with a higher incidence of cerebral palsy and neurodevelopmental disorders (1).

Increasingly, the significance of gender-specific biological susceptibility to disease is being recognized in translational studies $(3,5,7,20,21)$. Although endogenous estrogen production is not initiated before P12 in rodents, the role of maternal gonadal hormones and neonatal metabolism of estrogens and androgens, suggest an important role in gender susceptibility (5-7). Neurons derived from female brains predominantly use the cytochrome caspase 3-dependent apoptotic pathway whereas male neurons tend to use caspase-independent apoptotic pathways (22). Mechanisms involving mitochondrial resistance to injury/recovery also might contribute $(20,23)$. Clinical studies (e.g., stroke and preterm neonatal white matter injury) also have demonstrated gender differences with greater HII in males $(1,21)$ although gender effects on perinatal brain injury remain less certain (7).

Gender-dependent brain responses to treatment have been observed in several studies of perinatal brain injury therapeutics. For example, female rat pups respond better to erythropoietin (7), hypothermia or copper/zinc superoxide dismutase (24); caspase inhibitors (e.g., 2-Iminobiotin) $(8,9) ; 17 \beta$-estradiol (5); and "rehabilitative programs (11)." To a lesser degree, improved therapeutic responses in male rodents after neonatal HII have been reported (10). In clinical studies, indomethacin reduced the incidence of intraventricular and parenchymal hemorrhage (associated with higher childhood verbal scores) in very-low-birth-weight boys compared to girls (25). Thus, we felt it critical to ascertain whether such differences extended to stem cell-mediated interventions. Our data suggest that they do not-an observation heretofore unexplored in the field of perinatal HII treated with stem cells of any type. We observed no gender-significant differences in the severity of injury, in whole body animal weights over time, or in $90 \mathrm{~d}$ mortality. Implicit in our findings is the suggestion that matching the gender of the donor hNSCs to the gender of the recipient is not critical (12-14). The exhaustively characterized hNSCs that we employed in these studies were derived from a female fetus, yet performed equally well in male and female recipient rodent brains. The absence of gender effects is clinically relevant, as our data suggest that if hNSC transplantation trials 
in human newborns begin, gender need not be considered in candidate screening.

\section{Iron Labeling and HII Volume/Behavior}

Iron, the most abundant metal ion in the brain, is important for central nervous system development and is found in high levels within the globus pallidus and striatum, regions frequently affected by HII $(26,27)$. Iron content/distribution in the adult brain is largely established in the neonatal period (26). Iron is a cofactor in numerous processes including oxidative metabolism, free radical formation, and synthesis of nucleotides, proteins, and myelin. Iron chelation in immature animals reduces lesion size and improves motor/behavioral activity (26). Conversely, increasing free iron increases superoxide production and impairs memory, behavior, and white matter damage (28).

We found no evidence that SPIO labeling of hNSCs altered HII volume (Figure $3 \mathbf{b}, \mathbf{d}$ ) or behavior. Our dose of Feridex resulted in $\sim 85 \%$ labeling efficiency (17), similar to others, but there are considerable variations $(29,30)$. To our knowledge, our findings are the first to demonstrate that iron labeling of stem cells does not increase ischemic injury $(30,31)$. One previous study demonstrated that intracerebral administration of iron oxide into normal adult rat brain did not result in neuropathological abnormalities (32). This observation is consistent with our previous study in which iron-labeled murine NSCs migrated rapidly and survived long term (>1 y) (17). It is also consistent with other reports that, for the most part, SPIO labeling imparts virtually no adverse effects on stem cell viability and function in vivo at currently used labeling doses $(30,31)$.

\section{hNSCs Effective Only in Mild/Moderate but Not in Severe HII}

Although the primary aim of this study was to examine gender effects, an incidental, yet important, observation was that lesion volumes in pups receiving hNSCs did not increase over the $90 \mathrm{~d}$ observation period in the mild and moderate severity groups (countering the natural history of such lesions) but were unable to blunt the increase of lesion volume in the severe injury group (Figure 3c). This result is consistent with the observations of the effects of therapeutic hypothermia in term newborns (33) and in rodent models (34). The likely explanation for this finding is that the primary therapeutic action of our undifferentiated and nongenetically manipulated hNSCs is neuroprotection, and that severe HII is not simply quantitatively different from mild/moderate HII but is also qualitatively different in that host cell death happens quickly and extensively so that significant neuroprotection is not feasible. The hope, however, will be that, because of their unique properties (35), including being genetically modified to express additional factors (e.g., antiapoptotic) and/or to be layered upon biomaterials and/or to be predifferentiated down particular neural lineages (e.g., bridging interneurons), that stem cells will nevertheless ultimately offer new therapeutic approaches to all grades of HII. While additional studies are required to confirm and extend these new findings, we provide data suggesting that male and female newborns with HII have an equal potential to benefit from treatment with hNSCs.

\section{METHODS}

All experimental protocols complied with federal and Loma Linda University Animal Health and Safety Committee regulations.

\section{Animal Model of HII}

HII of the left hemisphere resulted in 20 male and 25 female 10-d-old Sprague-Dawley rat pups (Harlan, Livermore, CA) surviving as previously described (17). Using an MRI-based RPSS, we categorized injuries as mild $(0.25-0.9 ; n=9)$, moderate $(1.0-2.49 ; n=29)$, or severe $(>2.5 ; n=7$; Table 1) (36). We divided groups into those treated with SPIO labeled $(n=19)$ vs. unlabeled $(n=26)$ hNSCs.

\section{Neurological Testing}

Neurological testing (circling, C-shaped lateral bending, limb flexion, tremors, convulsions, righting reflexes, and negative geotaxis), as in our previous studies (17), was done daily (P10-13) to assess functional motor impairment. Summed scores ranged from 0 to $60(60=$ most impaired).

\section{hNSC}

We used hNSCs that are designated as "HFB 2050" cells, a stable line of hNSCs isolated from the ventricular zone of a single female human fetal cadaver that had been propagated with mitogens under defined conditions $(37,38)$. These well-characterized, genomically, and phenotypically stable hNSCs have been used safely and effectively for more than $13 \mathrm{y}$ in rodent and primate models. Cultured hNSCs were labeled with SPIO particles in a manner virtually identical to that previously used with mouse NSCs (17). Freshly trypsinized hNSCs were incubated for $24 \mathrm{~h}$ with Feridex $(11.2 \mu \mathrm{g} / \mathrm{ml})$ prior to implantation.

hNSCs were implanted into the contralateral (right) lateral ventricle $3 \mathrm{~d}$ after HII, as previously described (17). Rat pups were anesthetized with $3 \%$ isoflurane in $100 \% \mathrm{O}_{2}$. A Hamilton syringe was inserted through a burr hole and into the brain parenchyma to the ventricle $\left(2 \mathrm{~mm},+20^{\circ}\right)$ and a $5 \mu \mathrm{l}$ suspension of $\sim 250,000 \mathrm{hNSCs}$ was infused (1 $\mu \mathrm{l} / \mathrm{min}$ for $5 \mathrm{~min}$ ). The needle was removed after $5 \mathrm{~min}$, the burr hole closed with bone wax, and the scalp sutured.

\section{Neuroimaging and Analysis}

T2-weighted and diffusion-weighted imaging were performed at 1,30 , and $90 \mathrm{~d}$ postinjury as reported previously (17) on a Bruker Avance 11.7 T MRI (1 d) and a larger-bore Bruker 4.7 T machine (30, $90 \mathrm{~d}$ ). Data were analyzed using hierarchal region splitting to rapidly and semiautomatically identify regions of lesions (39). Analyses included HII volume, total brain volume with HII volumes expressed as a percent of total brain volume.

We employed the same MRI and computational methods as reported previously to estimate volumes noninvasively of SPIOlabeled NSCs $(17,40)$. Location of hNSCs was not detectable in pups implanted with unlabeled hNSCs. Although MRI cannot necessarily differentiate viable from nonviable cells (31), serial increases in NSC volume likely represent viable cell replication, an algorithm which we previously published (17). hNSC volumes were normalized to brain volume and averaged across all animals for each group (gender and time).

\section{Behavioral Testing}

A wide variety of behavioral domains were assessed at 2.5 mo similar to that previously reported $(17,36)$. Tests included measures of activity (open field), learning and memory (cued and spatial water maze), anxiety (zero maze), and sensorimotor coordination (rotarod).

\section{Histology/Immunohistochemistry}

Following $90 \mathrm{~d}$, animals were euthanized, perfused transcardially, and their brains removed and postfixed. Serial $30 \mu \mathrm{m}$ sections (every 10th section) were stained $(0.1 \%$ cresyl violet acetate). Prussian blue staining for SPIO containing cells was performed (17).

Free-floating sections were blocked with $10 \%$ normal goat serum (Invitrogen, Carlsbad, CA) for $10 \mathrm{~min}$. Primary antibodies were diluted in $0.1 \mathrm{~mol} / \mathrm{l}$ phosphate-buffered saline containing $10 \%$ 
normal goat serum and $0.1 \%$ triton $\mathrm{x}-100$ and used at the following concentrations: rabbit anti-hGFAP, 1:200 (Abcam, Cambridge, MA); mouse anti-hNestin, 1:100 (Abcam); mouse anti-human cyclic nucleotide phosphodiesterase, 1:100 (Millipore, Temecula, CA); mouse anti-human nuclei, 1:100 (Acris Antibodies, Germany); and mouse anti-rat GFAP, 1:300 (Sigma-Aldrich, St Louis, MO). Secondary antibodies (1:1,000; Invitrogen) included: goat antirabbit AlexaFluor ( 488 or $568 \mathrm{~nm}$ ) or goat anti-mouse AlexaFluor (488 or $555 \mathrm{~nm}$ ). Sections were air dried and coverslipped with VectaShield anti-fade mounting media (Vector Labs, Burlingame, CA). Slides were stored at $4{ }^{\circ} \mathrm{C}$ and immunolabeled slides were scanned on a confocal microscope (Bio-Rad 1024; Bio-Rad, Hercules, CA). $\mathrm{Z}$-series of $10-12$ images were collected by stepping through $\sim 1 \mu \mathrm{m}$ sections for each tissue.

\section{Statistics}

Data analyses used an $\alpha$-level of 0.05 to define significance. In our behavioral tests, to avoid violating statistical assumptions regarding compound symmetry and sphericity, the reported values for every repeated-measures analysis reflect the conservative HuynhFeldt correction to the degrees of freedom (SPSS, Armonk, NY). Significant main and interaction effects were explored using Scheffe's post hoc tests. General activity levels and cued spatial learning were analyzed with repeated-measures ANOVAs, while swim speed and sensorimotor coordination analyses were performed using one-way ANOVAs. MRI data significance was tested using one-way or twoway ANOVAs followed by appropriate post hoc testing (SigmaPlot V11, San Jose, CA).

\section{STATEMENT OF FINANCIAL SUPPORT}

This study was supported by grants from the Department of Pediatrics, Loma Linda University (Loma Linda, CA), and the National Institute of Neurological Disorders and Stroke, National Institutes of Health (Bethesda, MD; grant 1RO1NS059770-01A2).

Disclosure: There is no conflict of interest to disclose.

\section{REFERENCES}

1. Johnston MV, Hagberg H. Sex and the pathogenesis of cerebral palsy. Dev Med Child Neurol 2007;49:74-8.

2. de Courten-Myers GM. The human cerebral cortex: gender differences in structure and function. J Neuropathol Exp Neurol 1999;58:217-26.

3. Arteni NS, Pereira LO, Rodrigues AL, Lavinsky D, Achaval ME, Netto CA. Lateralized and sex-dependent behavioral and morphological effects of unilateral neonatal cerebral hypoxia-ischemia in the rat. Behav Brain Res 2010;210:92-8.

4. Mayoral SR, Omar G, Penn AA. Sex differences in a hypoxia model of preterm brain damage. Pediatr Res 2009;66:248-53.

5. Nuñez J. Sex and steroid hormones in early brain injury. Rev Endocr Metab Disord 2012;13:173-86.

6. Hill CA, Fitch RH. Sex differences in mechanisms and outcome of neonatal hypoxia-ischemia in rodent models: implications for sexspecific neuroprotection in clinical neonatal practice. Neurol Res Int 2012;2012:867531.

7. Wen TC, Rogido M, Peng H, Genetta T, Moore J, Sola A. Gender differences in long-term beneficial effects of erythropoietin given after neonatal stroke in postnatal day-7 rats. Neuroscience 2006;139:803-11.

8. Nijboer CH, Groenendaal F, Kavelaars A, Hagberg HH, van Bel F, Heijnen CJ. Gender-specific neuroprotection by 2 -iminobiotin after hypoxiaischemia in the neonatal rat via a nitric oxide independent pathway. J Cereb Blood Flow Metab 2007;27:282-92.

9. Renolleau S, Fau S, Goyenvalle C, et al. Specific caspase inhibitor Q-VD$\mathrm{OPh}$ prevents neonatal stroke in P7 rat: a role for gender. J Neurochem 2007;100:1062-71.

10. Saucier DM, Yager JY, Armstrong EA. Housing environment and sex affect behavioral recovery from ischemic brain damage. Behav Brain Res 2010;214:48-54.

11. Tsuji M, Aoo N, Harada K, et al. Sex differences in the benefits of rehabilitative training during adolescence following neonatal hypoxia-ischemia in rats. Exp Neurol 2010;226:285-92.
12. Csete M. Gender issues in transplantation. Anesth Analg 2008;107:232-8.

13. Lecanu L. Sex, the underestimated potential determining factor in brain tissue repair strategy. Stem Cells Dev 2011;20:2031-5.

14. Pearce V. Stem cells and neuroprotection: understanding the players. Int J Mol Sci 2010;11:3288-97.

15. Borlongan CV, Weiss MD. Baby STEPS: a giant leap for cell therapy in neonatal brain injury. Pediatr Res 2011;70:3-9.

16. Titomanlio L, Kavelaars A, Dalous J, et al. Stem cell therapy for neonatal brain injury: perspectives and challenges. Ann Neurol 2011;70:698-712.

17. Obenaus A, Dilmac N, Tone B, et al. Long-term magnetic resonance imaging of stem cells in neonatal ischemic injury. Ann Neurol 2011;69:282-91.

18. Park KI, Himes BT, Stieg PE, Tessler A, Fischer I, Snyder EY. Neural stem cells may be uniquely suited for combined gene therapy and cell replacement: Evidence from engraftment of Neurotrophin-3-expressing stem cells in hypoxic-ischemic brain injury. Exp Neurol 2006;199:179-90.

19. Smith EJ, Stroemer RP, Gorenkova N, et al. Implantation site and lesion topology determine efficacy of a human neural stem cell line in a rat model of chronic stroke. Stem Cells 2012;30:785-96.

20. Leger PL, Bonnin P, Lacombe P, et al. Dynamic spatio-temporal imaging of early reflow in a neonatal rat stroke model. J Cereb Blood Flow Metab 2013;33:137-45.

21. Vannucci SJ, Hurn PD. Gender differences in pediatric stroke: is elevated testosterone a risk factor for boys? Ann Neurol 2009;66:713-4.

22. Zhu C, Xu F, Wang X, et al. Different apoptotic mechanisms are activated in male and female brains after neonatal hypoxia-ischaemia. J Neurochem 2006;96:1016-27.

23. Normann S, de Veber G, Fobker M, et al. Role of endogenous testosterone concentration in pediatric stroke. Ann Neurol 2009;66:754-8.

24. Bona E, Hagberg H, Løberg EM, Bågenholm R, Thoresen M. Protective effects of moderate hypothermia after neonatal hypoxia-ischemia: shortand long-term outcome. Pediatr Res 1998;43:738-45.

25. Ment LR, Vohr BR, Makuch RW, et al. Prevention of intraventricular hemorrhage by indomethacin in male preterm infants. J Pediatr 2004;145:8324.

26. Caputa M, Rogalska J, Wentowska K, Nowakowska A. Perinatal asphyxia, hyperthermia and hyperferremia as factors inducing behavioural disturbances in adulthood: a rat model. Behav Brain Res 2005;163:246-56.

27. Connor JR, Pavlick G, Karli D, Menzies SL, Palmer C. A histochemical study of iron-positive cells in the developing rat brain. J Comp Neurol 1995;355:111-23.

28. de Lima MN, Polydoro M, Laranja DC, et al. Recognition memory impairment and brain oxidative stress induced by postnatal iron administration. Eur J Neurosci 2005;21:2521-8.

29. Hsiao JK, Tai MF, Chu HH, et al. Magnetic nanoparticle labeling of mesenchymal stem cells without transfection agent: cellular behavior and capability of detection with clinical $1.5 \mathrm{~T}$ magnetic resonance at the single cell level. Magn Reson Med 2007;58:717-24.

30. Cromer Berman SM, Kshitiz, Wang CJ, et al. Cell motility of neural stem cells is reduced after SPIO-labeling, which is mitigated after exocytosis. Magn Reson Med 2013;69:255-62.

31. Cromer Berman SM, Walczak P, Bulte JW. Tracking stem cells using magnetic nanoparticles. Wiley Interdiscip Rev Nanomed Nanobiotechnol 2011;3:343-55.

32. Muldoon LL, Sàndor M, Pinkston KE, Neuwelt EA. Imaging, distribution, and toxicity of superparamagnetic iron oxide magnetic resonance nanoparticles in the rat brain and intracerebral tumor. Neurosurgery 2005;57:785-96; discussion 785-96.

33. Shankaran S, Pappas A, Laptook AR, et al.; NICHD Neonatal Research Network. Outcomes of safety and effectiveness in a multicenter randomized, controlled trial of whole-body hypothermia for neonatal hypoxicischemic encephalopathy. Pediatrics 2008;122:e791-8.

34. Sabir H, Scull-Brown E, Liu X, Thoresen M. Immediate hypothermia is not neuroprotective after severe hypoxia-ischemia and is deleterious when delayed by 12 hours in neonatal rats. Stroke 2012;43:3364-70.

35. Kang KS, Trosko JE. Stem cells in toxicology: fundamental biology and practical considerations. Toxicol Sci 2011;120:Suppl 1:S269-89. 
36. Recker R, Adami A, Tone B, et al. Rodent neonatal bilateral carotid artery occlusion with hypoxia mimics human hypoxic-ischemic injury. J Cereb Blood Flow Metab 2009;29:1305-16.

37. Lee IS, Jung K, Kim M, Park KI. Neural stem cells: properties and therapeutic potentials for hypoxic-ischemic brain injury in newborn infants. Pediatr Int 2010;52:855-65.

38. Redmond DE Jr, Bjugstad KB, Teng YD, et al. Behavioral improvement in a primate Parkinson's model is associated with multiple homeostatic effects of human neural stem cells. Proc Natl Acad Sci USA 2007;104: 12175-80.

39. Ghosh N, Recker R, Shah A, Bhanu B, Ashwal S, Obenaus A. Automated ischemic lesion detection in a neonatal model of hypoxic ischemic injury. J Magn Reson Imaging 2011;33:772-81.

40. Ghosh N, Yuan X, Turenius CI, et al. Automated core-penumbra quantification in neonatal ischemic brain injury. J Cereb Blood Flow Metab 2012;32:2161-70. 